\title{
Originalien
}

\section{Fehlerquote bei der ICD-Verschlüsselung und Realisierbarkeit der Pflege-Personalregelung des Gesundheitsstrukturgesetzes}

\author{
A. König, R. Rau und P. C. Scriba \\ Medizinische Klinik (Direktor: Prof. Dr. P. C. Scriba), Klinikum Innenstadt der Universität München
}

Seit 1. 1. 1993 ist auf den Erhebungsbögen der Pflege-Personalregelung des Gesundheitsstrukturgesetzes die Angabe des ICD-Schlüssels (ICD = »international classification of diseases«) der Hauptdiagnose gesetzlich vorgeschrieben. Um die Fehlerquote bei dieser Verschlüsselung und deren Ursachen zu analysieren, wurden für das 1. Halbjahr 1993 die Daten der Pflege-Personalregelung erfaßt und die ICDSchlüssel mit den korrespondierenden Einträgen des Diagnosenarchivs abgeglichen. Bei 671 der 2308 überprüften Erhebungsbögen (29\%) wurden Fehler bei der Verschlüsselung festgestellt. Besonders hoch war die Fehlerquote in den Bereichen Kardiologie (39\%), Angiologie (37\%), Nephrologie (36\%) und Neurologie $(35 \%)$. Allerdings stammten die falschen Diagnosen in der Kardiologie und Nephrologie zu einem hohen Prozentsatz aus demselben Diagnosenteilbereich. - Im Sinne der Qualitätssicherung müssen die an der Verschlüsselung beteiligten Ärzte über die negativen Folgen für die Personalausstattung umfassend informiert werden; die Verschlüsselung muß von erfahrenen Kollegen durchgeführt, klinikintern überprüft und, falls technisch möglich, mit dem Diagnosenarchiv abgeglichen werden. Die Erfassung nur einer Hauptdiagnose ist bei multimorbiden Patienten nicht sinnvoll.

\author{
Frequency of errors in ICD coding in \\ relation to the implementation of \\ regulations for nursing personnel \\ provisions as prescribed in the \\ (German Federal Government's) \\ Health Organisation Law
}

Since 1 January, 1993, it has been a legal requirement to enter, for identification of the main diagnosis, the appropriate code from the International Classification of Diseases (ICD) on the documentation forms of the Nursing Personnel Regulation as prescribed under the (German Federal Government's) Health Organization Law. This study was undertaken to analyse the frequency of errors in this coding and the reasons for it. The data of the Nursing Personnel Regulations during the first 6 months of 1993 were compared with the corresponding entries in the diagnostic archives. In 671 of 2308 analysed forms $(29 \%)$ coding errors were discovered. The rate of errors was especially high in the areas of cardiology (39\%), angiology (37\%), nephrology (36\%) and neurology $(35 \%)$. However, a high percentage of the wrong codings for cardiological and nephrological diseases were still within the same diagnostic field. These findings indicate that, to ensure quality, those doctors who do the coding should be fully informed about the potential consequences regarding staffing of such errors. All coding must be done by experienced doctors, be locally checked and, if technically possible, compared with the diagnostic archive entries. Also, the registration of only one main diagnosis is not sensible in the case of patients with multi-organ disease.

telt. Diese auf den ersten Blick nur für den Pflegedienst bedeutsame Neuregelung tangiert den ärztlichen Bereich aufgrund der Tatsache, daß auf den zur Dokumentation in der Anlage 5 der Pflege-Personalregelung vorgeschriebenen Patienten-Erhebungsbögen auch der ICDSchlüssel der Hauptdiagnose anzugeben ist (ICD = »international classification of diseases $\ll$ )

Damit erhält die Arbeitsgemeinschaft der Spitzenverbände der Krankenkassen, an die die Erhebungsbögen weiterzuleiten sind, einen umfassenden Datenbestand, dessen weitere, über die Berechnung der
Dtsch. med. Wschr. 119 (1994), 755-759

(c) Georg Thieme Verlag Stuttgart · New York 
Porsonalstellen hinausgehende Analyse in der PflegePersonalregelung ausdrücklich vorgesehen ist: »Die Arbeitsgemeinschaft kann die Schlüssigkeit der Zuordnung der Patienten zu den Pflegestufen prüfen und einen Vergleich der Krankenhäuser untereinander vornehmen« (\$ 5 [3]). Da »Prüfung« und »Vergleich« im wesentlichen erst im Kontext der Hauptdiagnose ermöglicht werden und davon für die einzelnen Kliniken weitreichende finanzielle Folgen zu erwarten sind, erhält der ICD-Schlüssel einen so entscheidenden Stellenwert, daß dessen Richtigkeit, die stillschweigend vorausgesetzt wird, aber keineswegs sichergestellt ist, von größter $\mathrm{Be}$ deutung ist.

Nachdem an der Medizinischen Klinik seit 1987 ein patientenbezogenes Archiv aller Entlassungsdiagnosen gepflegt wird, bot sich die Möglichkeit, die auf den Patientenerhebungsbögen eingetragenen ICD-Schlüssel mit den entsprechenden Einträgen des Diagnosenarchivs abzugleichen. Ziel der hier vorgestellton Arbeit war es somit, die Richtigkeit dieser Verschlüsselung zu überprüfen, die aufgetretenen Fehler zu analysieren und Verbesserungsvorschläge zu erarbeiten. Zuvor mußten das Organisationsmodell und die Software zur Erfassung der Daten der Anlage 5 bereitgestellt werden.

\section{Methodik}

\section{Klinisches Umfeld}

Die Medizinischo Klinik des Klinikums Innonstadt der Ludwig-Maximilians-Universität München ist eine internistische Klinik mit insgesamt 181 stationären Betten, die sich auf acht Stationen verteilen. Eingeschlossen sind eine der Klinik angegliederte neurologische Spezialeinheit (das Friedrich-Baur-Institut, 19 Betten), eine Intensivstation (13 Betten), cine Tagesklinik, cine Zentrumsdialyse und mehrere Spezialambulanzen. Im Jahr 1992 wurden 6024 Patienten stationär behandolt. Die mittlere Liegezeit lag bei 10,2 Tagen.

\section{Archiv der Entlassungsdiagnosen}

Das Programmsystem, mit dem seit 1987 die Lintlassungsdiagnosen der stationären Patienten erfaßt und statistisch aufboreitet werden, wurde bercits an anderer Stelle ausführlich dargestellt (6). Kurz zusammengefaßt werden von dem für das Diktat verantwortlichen Arzt die in den Entlassungsberichten angegebenen Diagnosen verschlüsselt und die IC:D-Schlüssel im Entlassungsbrief aufgeführt. Zur Klassifikation wird eine an der Medizinischen Universität zu lü̈beck entwickelte Modifikation des ICD- $9(7,8)$ vorwendet. Die Diagnoseschlüssel (maximal 10) worden nach Eintreffen dor Krankenakte im Archiv vom dortigen Personal nach syntaktischer und semantischer Prüfung in das System eingegeben. Gleichzeitig werden dic sonstigen Basisdaten des stationären Aufenthaltes aus dem Verwaltungsrechner kopiert und dem Satz der Diagnoseschlüssel zugeordnet.

In Übereinstimmung mit den Empfehlungen der deutschen Gesellschaft für medizinische Dokumentation, Informatik und Statistik e. V. (2) und der Einleitung zur ICD (4) wurde dabei die Iauptdiagnose als die Haupterkrankung festgelegt, die während des Krankenhausa ufenthaltes im wesentlichen behandelt oder untersucht wurde. Falls keine Diagnose gestellt wurde, muß statt dessen das Hauptsymptom oder die schwerwiogendste Gesundheitsstörung gewählt werden. Dio mit der Verschlüsselung betrauten Kollegen wurden angehal ten, dio IIauptdiagnose joweils an die erste Stelle zu setzen.

\section{Erfassung und Bearbeitung der Patienten-Erhebungsbögen für die Pflege-Personalregelung}

Die Angaben auf den Erhebungsbögen wer den mit Ausnahme des ICD-Schlüssels vom Pflegepersonal nach entsprechender Einführung durch die Pflegedienstleitung - handschriftlich eingetragen bzw. Tag für Tag fortgeschrieben. An Stelle der »Akten-Nr. « wird ein von der Verwaltung bereitgestelltes maschinenlesbares Strichcodeetikett aufgebracht. Zum Zeitpunkt der Entlassung muß ein auf Station zuständiger Arzt den ICD-9-Schlüssel der Hauptdiagnose festlegen und eintragen. Die Bögen werden dann der Pflegedienstleitung zugeleitet und kommen von dort - nach einer ersten groben Prüfung auf Vollständigkeit - in die EDV-Abteilung.

Zur Eingabe der Daten wurde ein maskengesteuertes System entwickelt, mit dessen Hilfe der Inhalt der Erhebungsbögen in zwei Arbeitsschritten erfaßt wird: Im ersten Schritt werden die »Kopfdaten« erhoben: Dazu wird zunächst das Etikett mit der Aufnahmenummer mit Hilfc einer Lesepistole gelesen. Wurde bereits früher ein Bogen mit der entsprechenden Aufnahmenummer erfaßt, dann werden die zugehörigen Daten präsentiert und können verändert oder ergänzt werden. Im Falle einer neuen Aufnahmenummer werden die bereits im Verwaltungsrechner gespeicherten Daten (Aufnahmetag, Entlassungstag, Altersstufe) von dort via Rechnerkopplung angefordert und in den Datensatz übernommen. Diskrepante Angaben auf den Erhebungsbögen können an dieser Stelle sofort erkannt und die Bögen zur Berichtigung zurückgesandt werden. Danach muß der ICD-Schlüssel der Hauptdiagnose eingegeben werden. Die noch fehlenden technischen Angaben (vollstationäre Bchandlung, tagesklinische Bchandlung, Aufnahme von außen) sind mit den am häufigsten vorkommenden Angaben vorbelegt und müssen nur bei Abweichungen von Hand korrigiert werden. Im zweiten Schritt werden für jeden der Behandlungstage, die sich aus dem Aufnahme- und Entlassungstag ergeben, die Pflegestufen »A« und $» \mathrm{~S} \ll$ angefordert. Der Zeitaufwand für die Erfassung cines Bogens beträgt nach unseren bisherigen Erfahrungen - abhängig von der Liegezeit - zwischen 1 und 2 Minuten, das heißt, für unsere Klinik sind dafür pro Arbeitstag ungefähr $45 \mathrm{Minu}$ ten anzusetzen.

\section{Abgleich der ICD-Schlüssel}

Zum Abgleich wurde cin eigenes Prüfprogramm entwickelt: Übor einen variablen Zeitraum hinweg werden sämtliche Einträge des Diagnoscnarchives eingelesen und mit Hilfo der Aufnahmenummer für jedon Eintrag der korrespondierende Eintrag in der Datei der Patientenerhebungsbögen aufgesucht. Falls vorhanden, wird geprüf, ob der auf dem Erhebungsbogen angegebene ICD-Schlüssol der Hauptdiagnose unter den im Diagnosenarchiv abgelegten ICDSchlüsseln vorkommt. Eine falsche Verschlüsselung im Sinne der Untersuchung liegt dann vor, wenn die auf dem Patientenerhebungsbogen der PPR angegebene Hauptdiagnose im Archiv der Entlassungsdiagnosen überhaupt nicht vorkommt. Das bloße Abweichen der Hauptdiagnosen wird nicht als Fehler gewertet, da auch eine Nebendiagnose für den Pflegeaufwand von entscheidender Bedoutung sein kann. Für jeden fehlerhaften Eintrag wird ein Fohlerprotokoll ausgegcben, das die Station, die Aufnahmenummer, den ICD-Schlüssel des Patientenerhebungsbogens und alle ICD-Schlüssel des korrespondierenden Eintrags des Diagnosenarchivs umfaßt. 


\begin{tabular}{l|c|c|c}
\hline & $\begin{array}{c}\text { Anteil nicht überein- } \\
\text { stimmender } \\
\text { ICD-Schlüssel } \\
\text { innerhalb des } \\
\text { Teilbereichs (\%) }\end{array}$ & $\begin{array}{c}\text { Anteil bereichs- } \\
\text { konformer Schlüssel } \\
\text { an den nicht überein- } \\
\text { stimmenden Schlüsseln } \\
(\%)\end{array}$ & $\begin{array}{c}\text { Anteil der Schlüssel } \\
\text { dieses Teilbereichs am } \\
\text { Gesamtbestand aller } \\
\text { Patientenerhebungs- } \\
\text { bögen (\%) }\end{array}$ \\
\hline kardiologische Krankheiten & 39,1 & 88 & 35,7 \\
angiologische Krankheiten & 37,0 & 53 & 4,3 \\
nephrologische/urologische Krankheiten & 36,6 & 80 & 5,3 \\
neurologische Krankheiten & 35,1 & 56 & 8,8 \\
infektiöse Krankheiten & 27,4 & 57 & 2,7 \\
hämatologische Krankheiten & 25,0 & 66 & 1,4 \\
Hypertonie und rheumatische Krankheiten & 22,1 & 47 & 2,9 \\
Kollagenosen & 20,8 & 33 & 2,1 \\
Gastroenterologische Krankheiten & 20,2 & 66 & 4,9 \\
pneumologische Krankheiten & 16,3 & 29 & 4,0 \\
bösartige Krankheiten & 14,7 & 69 & 13,9 \\
endokrinologische Krankheiten & 10,3 & 64 & 9,3 \\
nicht-internistische Krankheiten & 35,8 & & 4,7 \\
\hline mittlere Fehlerquote insgesamt & 29,0 & & \\
\hline
\end{tabular}

Tab. 1 Aufschlüsselung der ICDSchlüssel der Hauptdiagnosen in den Erhebungsbögen der Pflege Personalregelung nach einzelnen Teilbereichen

\section{Ergebnisse}

Im ersten Halbjahr 1993 wurden an der Medizinischen Klinik 3109 Patienten stationär aufgenommen. Davon wurden bis zum Tag der Auswertung für 2896 (93\%) Krankheitsfälle die Entlassungsdiagnosen im Diagnosenarchiv eingetragen. Es fehlen die Patienten, für die bis dahin noch kein endgültiger Entlassungsbericht vorlag. Im gleichen Zeitraum wurde für 2593 Krankheitsfälle (83\%) ein Patientenerhebungsbogen erstellt und im System erfaßt. Hier fehlen vor allem die Patienten der Intensivstation, für die die PPR nicht anzuwenden ist.

Von diesen 2593 Erhebungsbögen konnten mit Hilfe der Aufnahmenummer $2308(89 \%)$ dem entsprechenden Eintrag im Diagnosenarchiv zugeordnet und die ICD-Schlüssel abgeglichen werden. Es zeigte sich, daß die ICD-Schlüssel der Hauptdiagnose der PPR bei 671 Einträgen (29\%) im Archiv der Entlassungsdiagnosen nicht gefunden wurden und damit die Verschlüsselung als fehlerhaft gewertet werden muß.

In Spalte 1 der Tab. 1 wird der prozentuale Anteil nicht übereinstimmender ICD-Schlüssel aufgeschlüsselt nach Teilbereichen absteigend sortiert aufgeführt; man erkennt, daß dieser Anteil zwischen $10 \%$ und $39 \%$ liegt. Die falschen ICD-Schlüssel aus dem Bereich der Kardiologie, Angiologie, Nephrologie und Neurologie liegen deutlich über dem Mittelwert von 29\%.

Eine eingehende Analyse der Fehlerprotokolle ergab, daß bei einer großen Zahl der im obigen Sinne falsch kodierten ICD-Schlüssel eine differenziertere Betrachtung möglich ist. Die in Tab. 2 aufgeführten Beispiele sollen dies erläutern.
Tab. 2 Beispiele für abweichende Hauptdiagnosen im Erhebungsbogen der Pflege-Personalregelung (PPR) und im Diagnosenarchiv

\begin{tabular}{c|l|l}
\hline Patient & PPR-Diagnose & Diagnosenarchiv \\
\hline A & Angina pectoris & $\begin{array}{l}\text { koronare Herzkrankheit, Mitralinsuffizienz, } \\
\text { Aortenklappenfehler }\end{array}$ \\
B & Angina pectoris & Hypothyreose, Mitralklappenfehler \\
C & Ulcus duodeni & Ulcus ventriculi \\
\hline
\end{tabular}

Sicher ist bei Patient A und C die abweichende Verschlüsselung weniger gravierend $\mathrm{zu}$ bewerten als bei Patient B. Wir nennen deshalb einen auf dem Patientenerhebungsbogen falsch kodierten ICD-Schlüssel »bereichskonform «, wenn sich unter den im Diagnosenarchiv eingetragenen ICD-Schlüsseln des gleichen Krankheitsfalles mindestens ein Eintrag aus demselben Teilbereich findet. Der Anteil der bereichskonformen ICD-Schlüssel an den falsch kodierten ICD-Schlüsseln der PPR ist in Spalte 2 der Tab. 1 aufgeführt. Es zeigt sich, daß dieser Anteil bei Krankheitsfällen aus dem kardiologischen und nephrologischen Teilbereich besonders hoch ist, da $\beta$ also in diesen Bereichen die abweichenden ICD-Schlüssel in gewissem Sinne »richtiger « gewertet müssen als bei den übrigen Bereichen.

In Spalte 3 von Tab. 1 wird der Anteil der ICD-Schlüssel aus den einzelnen Teilbereichen am Gesamtbestand angegeben. Er entspricht in etwa der aus früheren Jahren bekannten Verteilung (5) der Hauptdiagnosen des Diagnosenarchives.

Tab. 3 gibt den Anteil abweichender ICDSchlüssel für die einzelnen bettenführenden Stationen wieder. Dabei fällt das schlechte Abschneiden der Stationen F und I besonders ins Auge - ein Umstand, der ebenfalls zu diskutieren ist. 
Tab. 3 Anteil nicht übereinstimmender ICD-Schlüssel pro Station

\begin{tabular}{c|c}
\hline Station & Anteil (\%) \\
\hline A & 15 \\
B & 26, \\
C & 25 \\
D & 30 \\
E & 19 \\
F & 56 \\
G & 18 \\
H & 11 \\
I & 38
\end{tabular}

\section{Diskussion}

\section{Realisierbarkeit der Bestimmungen der Pflege-Personalregelung}

Unsere bisherigen Erfahrungen ergeben, daß die in der Pflege-Personalregelung des Gesundheitsstrukturgesetzes vorgeschriebene Erfassung der Daten mit vertretbarem Aufwand möglich ist: Die manuelle Erfassung mit Hilfe eines geeigneten System dauert pro Krankheitsfall zwischen 1 und 2 Minuten. Die Zeit, die für die Eingruppierung eines Patienten in die Pflegestufen »A « und »S « täglich aufzuwenden ist, liegt nach Auskunft mehrerer Stationsschwestern bei rund 5 Minuten. Dieser Zeitaufwand kann durch organisatorische Maßnahmen positiv beeinflußt werden, wenn zum Beispiel der Pflegeaufwand kontinuierlich dokumentiert wird und die Eingruppierung im Rahmen einer Pflegevisite oder-Übergabe erfolgt. Im Lauf der Zeit kommt es zu einer weiteren Beschleunigung, da die Pflegekräfte mit den Einordnungsmerkmalen aus den Anlagen 3 und 4 der Pflege-Personalregelung besser vertraut werden. Eine wesentliche Verminderung des globalen Zeitaufwandes läßt sich nur durch den Wegfall von Schreibarbeit oder durch Beschleunigung der EDV-mäßigen Erfassung der Daten erreichen.

Zwei Lösungsmöglichkeiten bieten sich an:

1. Pflegestufen werden direkt von den Pflegekräften in ein EDV-System eingegeben. Damit würde für das Pflegepersonal keine Mehrarbeit entstehen, da die handschriftliche Dokumentation entfallen könnte. Auch die vielzitierte »Schwellenangst « vor dem Medium Computer läßt sich, wie vergleichbare Ansätze zeigen (3), überwinden. Es ist jedoch die Ausstattung der Stationen mit geeigneter Hardware (Personalcomputer oder »notepads «) erforderlich - eine Investition, die gerade im Hinblick auf die noch zu erwartenden Aufgaben (unter anderem Leistungserfassung, Fallpauschalen, Sonderentgelte, Abteilungspflegesätze) sicherlich ohnehin bald unumgänglich sein wird.

2. Die zweite Möglichkeit läßt sich schneller und kostengünstiger realisieren: Umstellung der Erfassungsbögen auf maschinell lesbare Markierungsbelege. Dadurch wird die Datenerfassung ohne Mehraufwand und ohne wesentliche Kosten deutlich reduziert. Diesen Lösungsweg, der bereits kommerziell angeboten wird, werden wir voraussichtlich in Kürze beschreiten.

\section{Fehlerhafte ICD-Verschlüsselung}

Eine exakte Einzelüberprüfung der auf den Patientenerhebungsbögen eingetragenen ICDSchlüssel ist nicht mit sinnvollem Aufwand durchführbar. Wir haben deshalb versucht, durch Abgleich der ICD-Schlüssel der PPR mit den im Archiv der Entlassungsdiagnosen gespeicherten Angaben Aussagen über deren Richtigkeit bzw. Konsistenz zu gewinnen. Dieser methodische Ansatz bietet sich an, da die im Archiv gespeicherten Diagnosenschlüssel erst zum Zeitpunkt des Entlassungsberichts, also nach Vorliegen aller Ergebnisse, festgehalten und vom zuständigen Oberarzt kontrolliert werden; diese Angaben sind damit sicher zuverlässiger als die meist »zwischen Tür und Angel» eingetragenen Schlüssel der Patientenerhebungsbögen.

Der Abgleich ergibt, daß der ICD-Schlüssel der auf den Erhebungsbögen angegebenen Hauptdiagnose bei unserer Untersuchung nur in etwa $70 \%$ mit einer der im Entlassungsbericht aufgeführten Diagnosen übereinstimmt. Nachdem in unserer Klinik die Verschlüsselung der Entlassungsdiagnosen seit 1987 eingeführt ist und damit vorausgesetzt werden darf, daß die Ärzte darin ausreichende Erfahrung besitzen, muß ein mindestens ähnlich fehlerhaftes Ergebnis auch aus anderen Häusern erwartet werden. Das ist um so beunruhigender, als den ICD-Schlüsseln bei der in der Pflege-Personalregelung vorgesehenen Auswertung der Erhebungsbögen sicher eine zentrale Rolle zukommen wird, deren finanziellen und personellen Folgen noch gar nicht genau abzusehen sind. Wie die Auswertung aussehen könnte, geht aus einer Arbeit von Scheinert und Wöhrmann (9) hervor, in der unter anderem die Patientenstruktur, die Behandlungsergebnisstruktur, die Leistungsintensität für Diagnostik und Therapie, die Pflegeintensität und die Verweildauerstruktur angeführt werden.

Aus unseren Ergebnissen und der Einzelanalyse des Fehlerprotokolles lassen sich zwar nicht alle Fehler erklären, aber häufige Fehlerquellen aufzeigen:

- mangelnde Motivation aufgrund unzureichender Information: Bei der gesetzlich vorgeschriebenen Einführung des Patientenerhebungsbogens wurden zwar die Pflegekräfte, nicht aber die zuständigen Ärzte ausreichend informiert. Letztere sahen sich nicht selten mit einem neuen Formular konfrontiert, dessen Bedeutung ihnen nicht klar und deshalb gleichgültig war. So ist es verständlich, daß oftmals ohne längeres Nachdenken, um der Pflicht Genüge zu tun, der Bogen ausgefüllt wurde und die eingetragenen ICDSchlüssel nur grob der tatsächlichen Hauptdiagnose entsprechen - eine Aussage, die sich auch am hohen Anteil bereichskonformer Schlüssel ablesen läßt. Der hohe Fehleranteil der kardiologischen Schwerpunktstation (Station F) soll hierfür als Beispiel gelten: Es wurde in fast 200 Fällen das Symptom »Angina pectoris« verschlüsselt; im endgültigen Bericht erscheint dann in fast allen Fällen die korrekte Diagnose »koronare Herzkrankheit« oder »Myokardinfarkt«.

- mangelnde Vertrautheit: Ungenügende Kenntnis des Schlüsselverzeichnisses führt dazu, daß - auch bei gutem Willen des verschlüsselnden Arztes - ein unschar- 
fer oder falscher ICD-Schlüssel eingetragen wird. Dadurch erklärt sich zum Teil der hohe Anteil falsch eingetragener Diagnosen auf Station I, einer Abteilung, in der aus organisatorischen Gründen die routinemäBige Pflege des Diagnosenarchives erst mit Beginn dieses Jahres eingeführt wurde und somit erst relativ kurze Zeit Erfahrungen mit dem Schlüsselwerk gesammelt wurden.

- ungünstiger Zeitpunkt: Der ICD-Schlüssel der Hauptdiagnose muß zum Zeitpunkt der Entlassung des Patienten ad hoc in den Patientenerhebungsbogen eingetragen werden. Jedem, der mit dem Ablauf der Stationsarbeit vertraut ist, ist klar, daß dieser Zeitpunkt relativ ungünstig liegt: Einerseits fällt er in eine Phase vermehrter Aktivitäten, in der Formalitäten notgedrungen rasch erledigt werden müssen. Zudem liegen in vielen Fällen noch nicht alle Ergebnisse vor, so daß die Hauptdiagnose tatsächlich nicht immer schon sicher feststeht. Beispiele hierfür sind unter anderem im onkologischen und hämatologischen Bereich zu finden, wenn die endgültige Histologie noch aussteht. Trotzdem raten wir davon ab, den Eintrag des ICD-Schlüssels in den Patientenerhebungsbogen auf einen wesentlich späteren Zeitpunkt zu verschieben, weil sonst die Gefahr, daß der Bogen ohne ICDSchlüssel bleibt, ansteigt.

- Schwierigkeiten bei der Wahl der Hauptdiagnose: Bei einem steigenden Anteil multimorbider Patienten wird der Begriff der Hauptdiagnose zunehmend fraglich. In vielen Fällen bestehen gesundheitliche Probleme so vielfältiger Art, daß die Festlegung auf eine Hauptdiagnose kaum eindeutig möglich ist. Daraus ergeben sich eine Reihe falscher Einträge. Beispielhaft ist hier der angiologische Bereich, wo wiederholt auf dem Erhebungsbogen eine angiologische Erkrankung angegeben wurde, während im Entlassungsbericht andere Begleitkrankheiten erscheinen.

\section{Möglichkeiten der Verbesserung}

Aus den genannten Fehlerquellen lassen sich einige naheliegende, aber wichtige Vorschläge zur Verbesserung im Sinne der Qualitätssicherung ableiten. Die wichtigste Voraussetzung ist unseres Erachtens die Steigerung der Motivation der beteiligten Ärzte durch genaue Information über die Hintergründe der angeordneten Maßnahmen. Nur wenn jedem wirklich klar ist, welche Folgen unexakte oder gar falsche Angaben haben können, ist damit zu rechnen, da $\beta$ sich ein Verantwortungsgefühl für diese Aufgabe entwickeln wird. Diese grundlegende Maßnahme gewinnt im Hinblick auf in $\mathrm{Zu}-$ kunft zu erwartende Dokumentationsaufgaben an zusätzlichem Gewicht.

Die ICD-Verschlüsselung darf nicht den jüngsten und unerfahrendsten Kollegen überlassen werden, sondern muß vom verantwortlichen Stationsarzt oder mit einem Prüfprogramm - ähnlich wie in unserer Studie - kontrolliert und gegebenenfalls korrigiert werden. Dazu ist die Anonymität des Patientenerhebungsbogens bis zu dessen Versand außer Hauses aufzuheben; andernfalls werden Korrekturen sehr erschwert. Falls die Daten per Datenträger übermittelt werden und die Bögen das Haus gar nicht verlassen, ist Anonymität unseres Erachtens nicht erforderlich.
Da den im Arztbrief aufgeführten Diagnosen die höhere Zuverlässigkeit zukommt, sollte bei den Krankenhäusern, die über ein EDV-unterstütztes Diagnosenarchiv verfügen, ein automatischer Abgleich der auf den Patientenerhebungsbögen angegebenen ICDSchlüssel mit dem Diagnosenarchiv erfolgen.

Eine deutliche inhaltliche Verbesserung ließe sich erzielen, wenn auf dem Patientenerhebungsbogen nicht nur die Angabe einer einzigen Hauptdiagnose, sondern die der drei oder vier wichtigsten Diagnosen möglich wäre. Damit könnte das angesprochene Dilemma des Begriffes der Hauptdiagnose umgangen und der Grund für den Aufenthalt exakter umrissen werden.

Daß die Fehlerquote durch die vorgeschlagenen Maßnahmen deutlich reduziert werden kann, belegen die niedrigen Fehlerquoten einiger unserer Stationen. Sollte wider Erwarten der hohe Anteil fehlerhafter Hauptdiagnosen von $29 \%$ nicht wesentlich reduziert werden können, wäre ernstlich zu fragen, ob solche Daten als Grundlage wichtiger Entscheidungen tauglich sind.

\section{Literatur}

1 Gesetz zur Sicherung und Strukturverbesserung der gesetzlichen Krankenversicherung (Gesundheitsstrukturgesetz) vom 21. Dezember 1992. Bundesgesundheitsblatt I (1992), $2266-2334$

2 Göhring, R., P. Haas, W. D. Hoffmann, R. Klar, W. Wilde, F. Win gert: Empfehlungen zur Dokumentation und Auswertung von Diagnosen in Krankenhäusern. Heft 8 der Schriftenreihe der Deutschen Gesellschaft für medizinische Dokumentation, Informatik und Statistik e. V. (Schattauer: Stuttgart-New York 1986).

3 Greil, A.: Notepads in Pflege und Medizin. Führen und Wirtschaften im Krankenhaus ( $\&$ w) 10 (1993), 246-251.

4 Internationale Klassifikation der Krankheiten, Verletzungen und Todesursachen (ICD) in der Fassung der vom Bundesminister für Jugend, Familie und Gesundheit herausgegebenen, 9. Revision (Kohlhammer: Köln-Stuttgart-Berlin-Mainz 1986).

5 Klinikum Innenstadt - Medizinische Klinik: Leistungsbericht 1992 (Eigendruck 1993).

6 König, A.: Data structure and computer program for fast interactive searches of clinical diagnoses. Med. Inform. 18 (1993), 45-51.

7 Mansky, T., P. C. Scriba, H. J. Friedrich, H. Fassl: Der Lübecker Diagnoseschlüssel. Klinikarzt 15 (1986), 1062.

8 Mansky, T., P. C. Scriba, H. Fassl, H. J. Friedrich: Diagnose-Verschlüsselung: wie und wozu? Dtsch. med. Wschr. 111 (1986), $1707-1708$

9 Scheinert, H. D., S. Wöhrmann: Die Pflege-Personalregelung Die Ersatzkasse 7 (1993), 277-285

Dr. med. Dipl.-Math. A. König, Dipl,-Ing. R. Rau,

Prof. Dr. P. C. Scriba

Medizinische Klinik

Klinikum Innenstadt

Ziemssenstr. 1

80336 München 